\title{
Analysis of the EEG dynamics of epileptic activity in gelastic seizures using decomposition in independent components
}

\author{
Alberto J.R. Leal $^{\mathrm{a}, \mathrm{b}, *}$, Ana I. Dias ${ }^{\mathrm{b}}$, José P. Vieira ${ }^{\mathrm{b}}$ \\ ${ }^{a}$ Department of Neurophysiology, Hospital Júlio de Matos, Av va do Brasil, No. 53, 1749-002 Lisbon, Portugal \\ ${ }^{\mathrm{b}}$ Department of Pediatric Neurology, Hospital Dona Estefânia, Lisbon, Portugal
}

Accepted 16 March 2006

\begin{abstract}
Objective: Gelastic seizures are a frequent and well established manifestation of the epilepsy associated with hypothalamic hamartomas. The scalp EEG recordings very seldom demonstrate clear spike activity and the information about the ictal epilepsy dynamics is limited. In this work, we try to isolate epileptic rhythms in gelastic seizures and study their generators.

Methods: We extracted rhythmic activity from EEG scalp recordings of gelastic seizures using decomposition in independent components (ICA) in three patients, two with hypothalamic hamartomas and one with no hypothalamic lesion. Time analysis of these rhythms and inverse source analysis was done to recover their foci of origin and temporal dynamics.

Results: In the two patients with hypothalamic hamartomas consistent ictal delta $(2-3 \mathrm{~Hz})$ rhythms were present, with subcortical generators in both and a superficial one in a single patient. The latter pattern was observed in the patient with no hypothalamic hamartoma visible in MRI. The deep generators activated earlier than the superficial ones, suggesting a consistent sub-cortical origin of the rhythmical activity. Conclusions: Our data is compatible with early and brief epileptic generators in deep sub-cortical regions and more superficial ones activating later.

Significance: Gelastic seizures express rhythms on scalp EEG compatible with epileptic activity originating in sub-cortical generators and secondarily involving cortical ones.

(C) 2006 International Federation of Clinical Neurophysiology. Published by Elsevier Ireland Ltd. All rights reserved.
\end{abstract}

Keywords: Epilepsy; Hypothalamus; Hamartoma; ICA; Gelastic; Seizures

\section{Introduction}

Gelastic seizures are a rare type of epileptic manifestation where laughter spells occur without a concomitant sense of joy. They are usually brief (less than $1 \mathrm{~min}$ ) and can occur several times daily. Historically, these seizures have been associated with hypothalamic hamartomas (List et al., 1958), and more recent invasive EEG recordings demonstrated the existence of paroxysmal activity limited to the hamartoma during such events (Munari et al., 1995). Nevertheless cases have been reported where no

\footnotetext{
* Corresponding author. Address: Department of Neurophysiology, Hospital Júlio de Matos, Ava do Brasil, No. 53, 1749-002 Lisbon, Portugal. Tel.: + 351 969851734; fax: +351 217819809 .

E-mail address: a.leal@netcabo.pt (A.J.R. Leal).
}

hypothalamic lesions could be found (Arroyo et al., 1993) and this suggested the existence of extra-hypothalamic generators.

In previous studies, we used source analysis techniques of the interictal spike activity of patients with hypothalamic hamartomas to demonstrate the existence of deep generators in the neighborhood of the lesion (Leal et al., 2002) and also that the EEG scalp topography can show frontal or temporal dominance depending on the particular anatomy of the connection of the hamartoma to the hypothalamus (Leal et al., 2003).

The scalp EEG recordings during the seizures have not been very informative (reviewed in Munari et al., 2000) because no clear paroxysmal activity is usually found and the poor signal to noise ratio of such recordings has prevented more detailed analysis. Nevertheless visual 
inspection of ictal EEG reveals the existence of rhythmical activity in the theta-delta range which could provide useful information on the dynamics of this type of epilepsy. In this study, we perform analysis of ictal EEG recordings in patients with gelastic seizures, trying to separate true epileptic activity from artifacts and gain a deeper understanding of the dynamics and possible localizations of the epileptic generators.

\section{Methods and subjects}

\subsection{Methods}

Seizures of three patients with gelastic epilepsy were recorded during long-term video-EEG monitoring (12 h) using setups with 32,30 and 21 scalp gold electrodes. Clinical data is presented in Table 1, and has been detailed previously for patients 2 (Leal et al., 2003) and 3 (Leal et al., 2002).

The sampling rate of EEG acquisition was $256 \mathrm{~Hz}$ and it was filtered with a high pass filter at $0.5 \mathrm{~Hz}$ and a low pass at $70 \mathrm{~Hz}$. All seizures were signaled by the patient or by an accompanying family member. The seizure onset time was determined by careful analysis of behavioral manifestations in the video recording. The appearance of high frequency scalp EMG activity on the EEG (Figs. 1(a) and 2(a)) resulting from a characteristic sudden agitation preceding the first laughter, or EEG desynchronization with increase of fast rhythms (Fig. 3(a)) were additional features used to determine seizure onset.

Seizures were segmented between -30 and $30 \mathrm{~s}$ in relation to seizure onset. All lasted less than a minute and most less than $30 \mathrm{~s}$. A total of eleven seizures were collected for patients 1 and 3 for each one of the two remaining patients. The segmented seizures were imported into the EEGLAB software (Delorme and Makeig, 2004) running in MatLab 6.1 (MathWorks), merged in a single dataset for each patient and submitted to Independent Component Analysis (ICA) for decomposition in independent components (Makeig et al., 2004) (Appendix A). Afterward we selected the components with rhythmical activity after seizure onset (Figs. 1(b)-3(b)) which represented all rhythmic activity in the theta-delta range in the raw EEG (Figs. 1(a)-3(a)). These components were submitted to a FFT analysis and the statistically significant changes at the different frequencies were color coded to produce a graphical representation of the spectral changes induced by the seizure, a so called event related spectral perturbation (ERSP) (Makeig, 1993). Significance of the changes was quantified using a Bootstrap method (Appendix A).
Table 1

\begin{tabular}{|c|c|c|c|}
\hline & Patient 1 & Patient 2 & Patient 3 \\
\hline Age & 9 & 6 & 2 \\
\hline Sex & $\mathrm{F}$ & M & $\mathrm{F}$ \\
\hline \multicolumn{3}{|l|}{ Epilepsy } & Delayed \\
\hline Age of onset & 9 years & 3 years & 1 week \\
\hline Seizure type & Gelastic & $\begin{array}{l}\text { Gelastic + partial } \\
\text { complex }\end{array}$ & $\begin{array}{l}\text { Gelastic + partial } \\
\text { complex }\end{array}$ \\
\hline Seizure freq & $\begin{array}{l}\text { Seizure free } \\
\text { with } \mathrm{AE}\end{array}$ & $\begin{array}{l}3-5 \text { daily } \\
\text { seizures }\end{array}$ & $\begin{array}{l}>10 \text { daily } \\
\text { seizures }\end{array}$ \\
\hline \multicolumn{4}{|l|}{ EEG } \\
\hline Interictal & $\begin{array}{l}\text { Frontal lobe } \\
\text { spikes }\end{array}$ & $\begin{array}{l}\text { Temporal lobe } \\
\text { spikes }\end{array}$ & $\begin{array}{l}\text { Temporal lobe } \\
\text { spikes }\end{array}$ \\
\hline Ictal & $\begin{array}{l}\text { Theta-delta } \\
\text { activity }\end{array}$ & Delta activity & $\begin{array}{l}\text { Beta }+ \text { delta } \\
\text { activity }\end{array}$ \\
\hline MRI & No hamartoma & $\begin{array}{l}\text { Hypoth. } \\
\text { hamartoma }\end{array}$ & $\begin{array}{l}\text { Hypoth. } \\
\text { hamartoma }\end{array}$ \\
\hline
\end{tabular}

Source analysis was performed on the spatial component of each rhythmical activity after seizure onset, using a spherical volume conduction model to determine the localization of the generator of each scalp rhythm (Appendix A).

\section{Results}

Seizures in the different patients showed a remarkable similarity, with early desynchronization of the EEG, which reduced in amplitude due to the disappearance of the alpha, theta and delta activities, followed seconds later by onset of slow rhythmic activity in the theta-delta frequency range. Such rhythms can be diffuse such as in patient 3 (Fig. 3(a)) or focalized such as in patient 1 (Fig. 1(a)). A typical increase in diffuse EMG activity and also movement artifacts is seen at seizure onset (Figs. 1(a) and 2(a)).

The ICA components with rhythmical activity are significantly reduced in number as compared with the number of EEG channels expressing such rhythms (Figs. 1(b)-3(b)) representing a significant convergence of information. These components express a dipolar type of distribution of the potential over the scalp (Figs. 1(c)-3(c)), suggesting that a focal source might be a good representation of the intracranial generators of such rhythms. Furthermore, no significant activity of these components is observable in the $30 \mathrm{~s}$ period preceding the seizure, suggesting that they represent a true ictal phenomena (Figs. $1(\mathrm{c})-3(\mathrm{c}))$.

The topography of ictal rhythmical components falls in one of two categories: type 1 has polarities on opposite sides of the head, compatible with a deep source; type 2 has

seizures. There is an increase at $2-3 \mathrm{~Hz}$, with an early onset in component 2 (left scale in $\mathrm{Hz}$, color scale $-25 \mathrm{~dB}$ blue an $+25 \mathrm{~dB}$ red; green is non-significant at the Bootstrap level of 1\%). (d) Best fit dipole solution for components 2 (left), 1 (middle) and 3 (right), with 1 SD confidence ellipsoids (Fuchs et al., 2004). A deep localization is seen for component 2 while the others have more superficial solutions. (For interpretation of the references to colour in this figure legend, the reader is referred to the web version of this article.) 
(a)

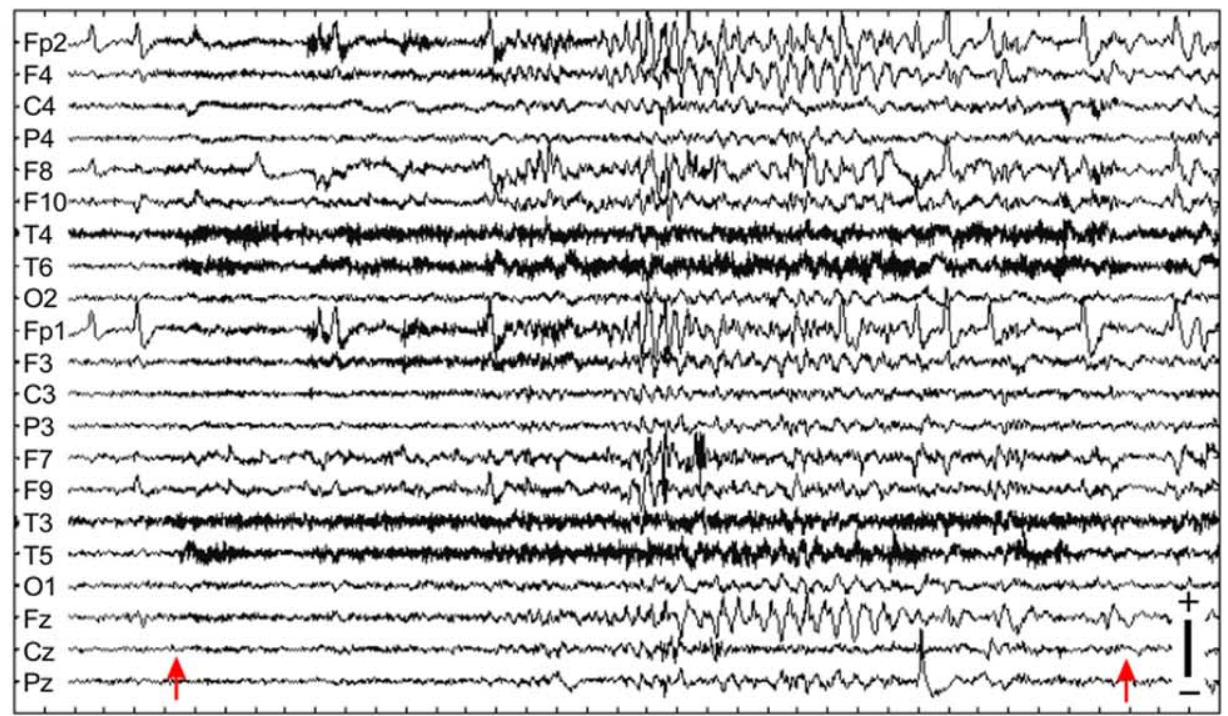

(b)
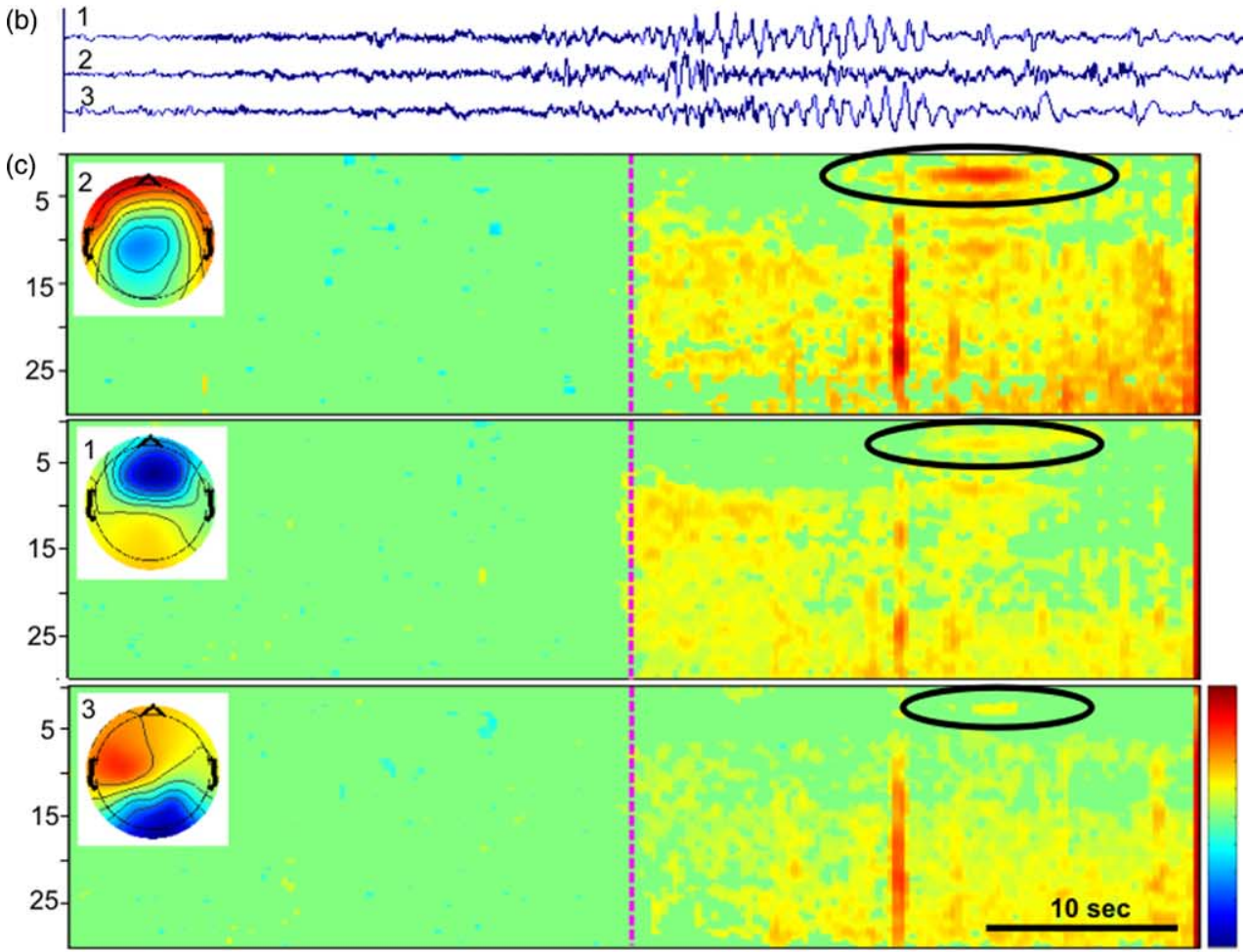

(d)
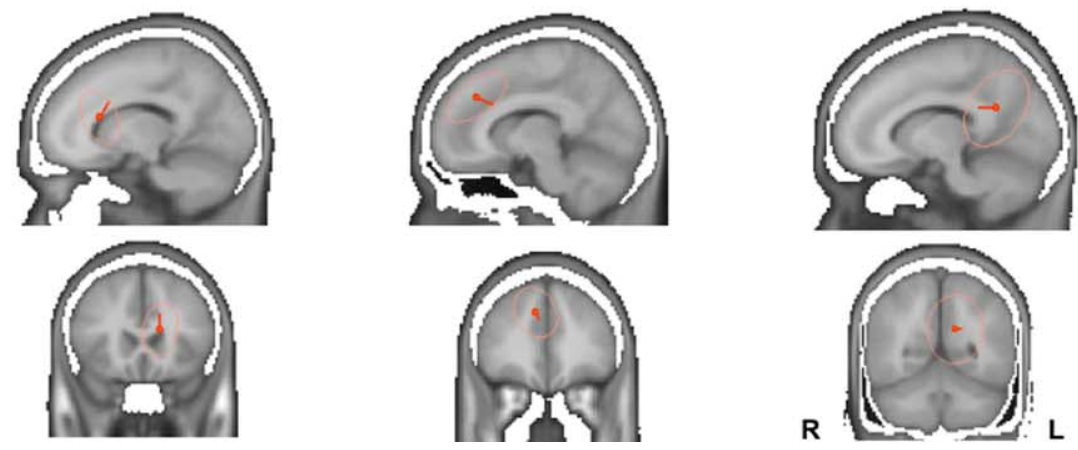

Fig. 1. (a) Representative EEG of a gelastic seizure for patient 1 (arrows signal seizure onset and offset). There are no spikes, but a rhythmical delta activity is seen over the frontal electrodes (time scale in seconds, amplitude scale is $500(\mathrm{~V})$. (b) Time course of ICA components with rhythmical activity during the gelastic event. (c) Event related spectral perturbation (ERSP) for the three ICA components between -30 and $+30 \mathrm{~s}$ centered on seizure onset, for 11 merged 


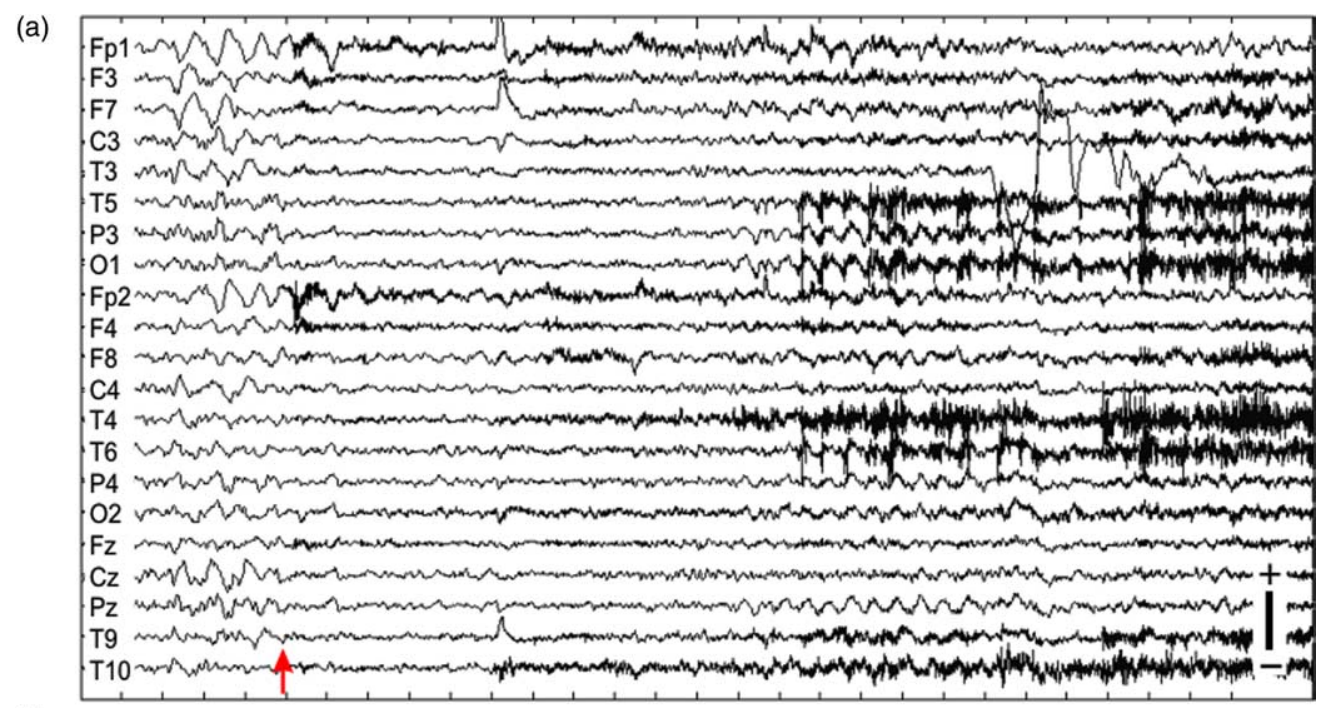

(b)
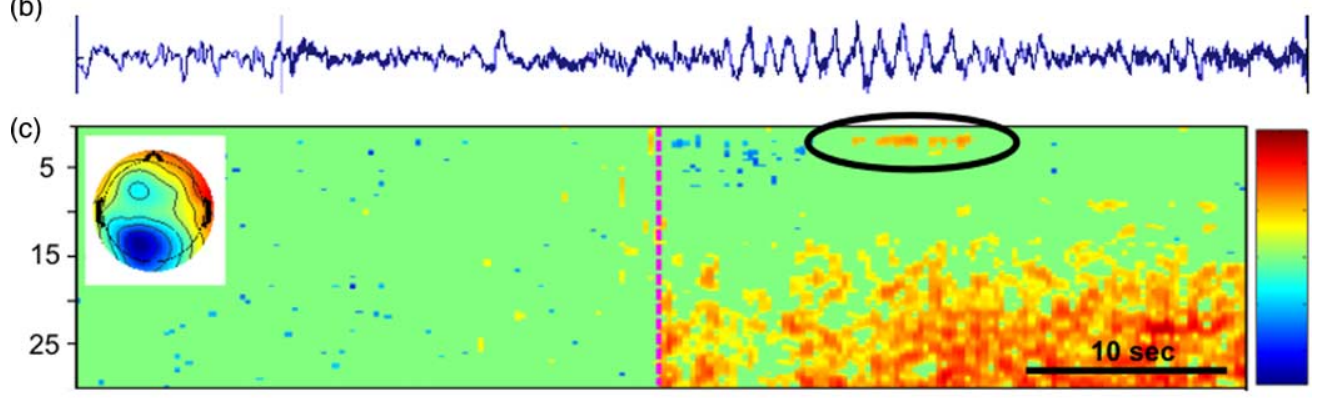

(d)
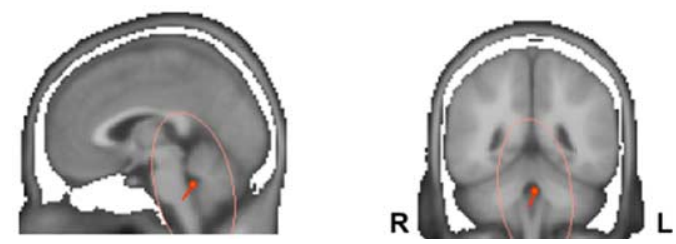

Fig. 2. (a) Representative EEG of a gelastic seizure for patient 2 (arrow signals seizure onset). There are no spikes, but a diffuse rhythmical delta activity is seen. (b) Time course of the only rhythmical ICA component obtained from the merged gelastic events. (c) ERSP for the ICA component, for three merged seizures. There is an increase in the 2-3 Hz frequency a few seconds after seizure onset (scales as in Fig. 1). (d) Best fit dipole solution for the ICA component, with the $1 \mathrm{SD}$ confidence ellipsoids. A deep posterior localization is obtained.

polarities closer together, typically in the same hemisphere, which is more compatible with superficial sources. All patients have one or more components of type 1 , but only patients 1 and 3 have components of type 2 .

The ERSP graphs demonstrate a clearly demarcated activity in the frequency range of $2-3 \mathrm{~Hz}$, and the early onset of such activity in type 1 components supports an earlier activation as compared to the ones of type 2 .

Source analysis of the different types of components leads to deep generators for type 1 and superficial ones for type 2 (Figs. 1(d)-3(d)). Adjusted dipoles for type 1 components lie in the deep frontal and thalamus-hypothalamus regions showing residual variances $(\mathrm{RV})$ of 3,9 and $9-11 \%$ for patients 1, 2 and 3, respectively. Localization of type 2 dipoles is superficial in the frontal, parietal or temporal lobes and the RVs are of 5-7\% for patient 1 and $20 \%$ for patient 3 .

\section{Discussion}

The main result of this study is that gelastic seizures produce rhythmical activity on the scalp EEG, of probable epileptic nature and with generators in the deep sub-cortical regions (thalamus/hypothalamus) and also in cortical areas.

Gelastic seizures typically do not exhibit clear spike activity (Munari et al., 2000) and the frequent coexistence of movement and muscular artifacts makes the identification of epileptic rhythms difficult. It has historically been assumed that no relevant information can be obtained about the seizure generators from scalp ictal recordings and this fact was attributed to the focal and deep localization of such generators (Munari et al., 1995). Despite this historical background, our analysis of the raw EEG of several seizure recordings persistently revealed the existence of small amplitude rhythmical activity not attributable to artifacts 
(a)

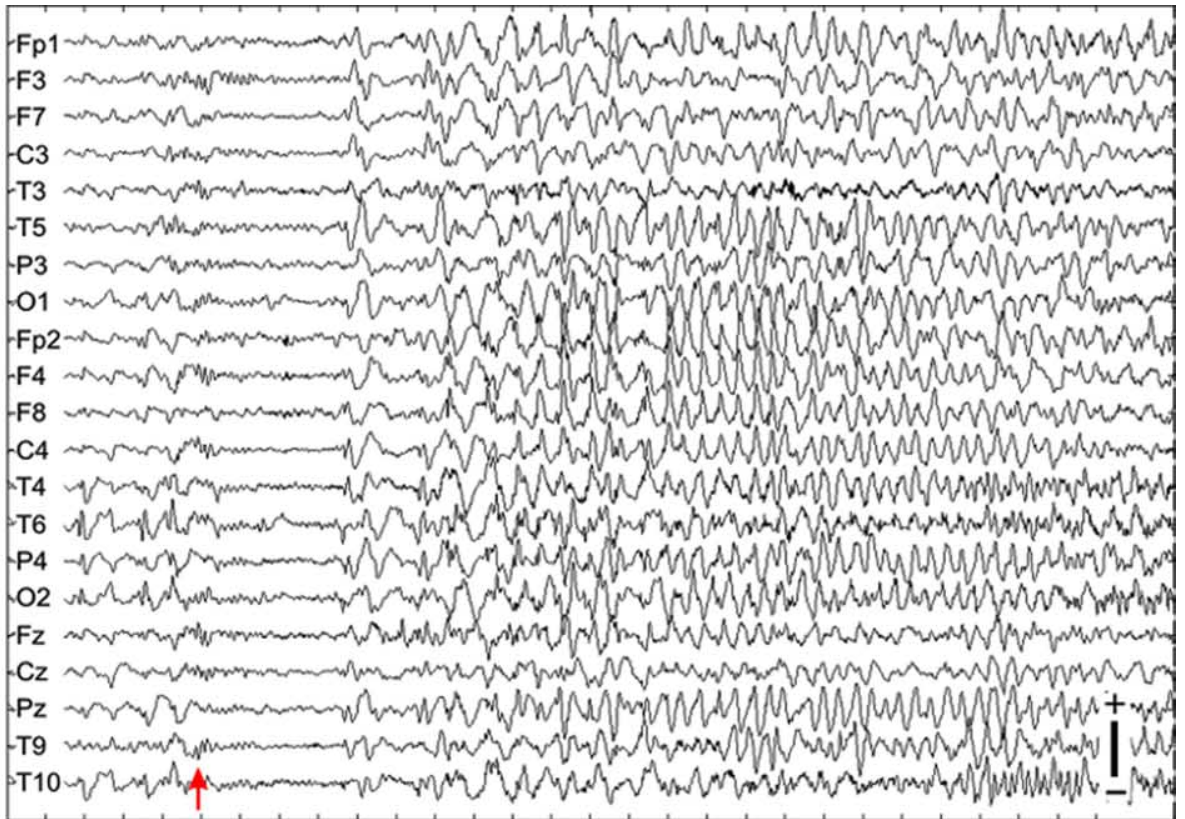

(b)

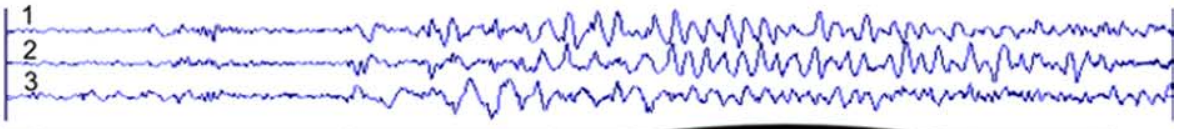

(c)

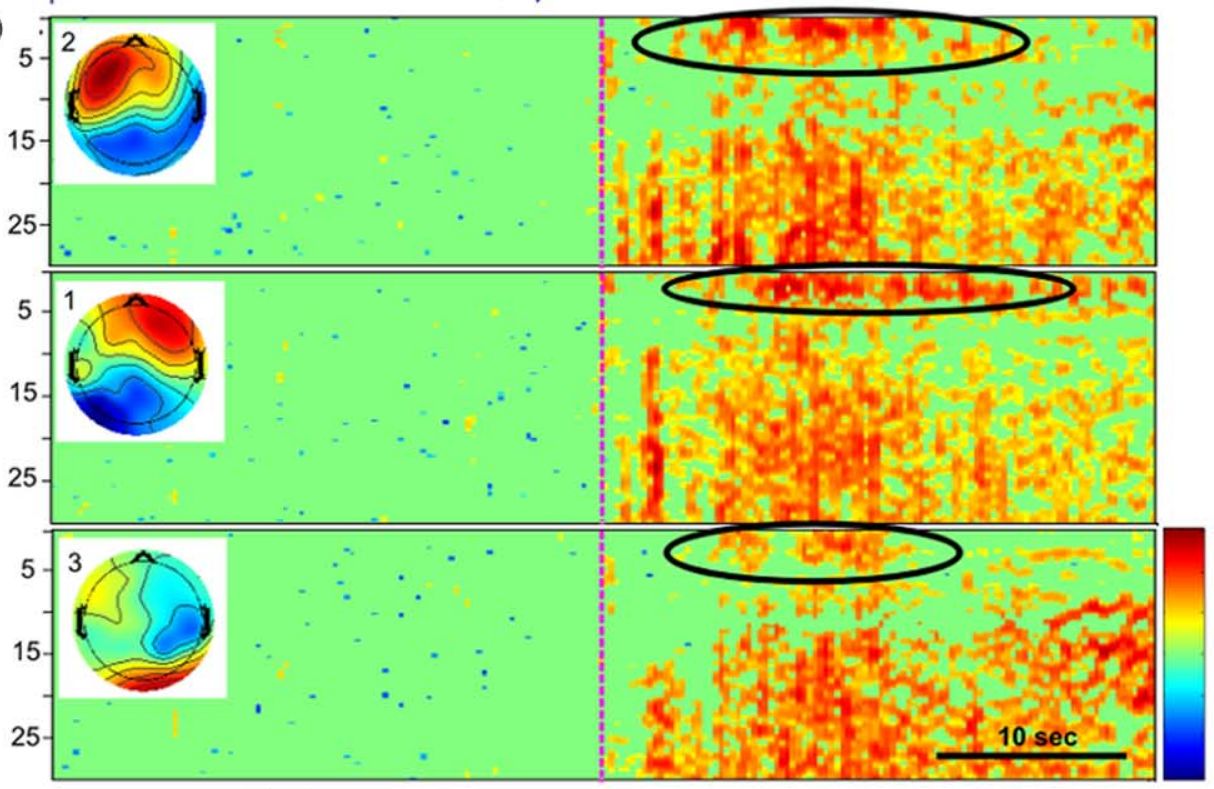

(d)
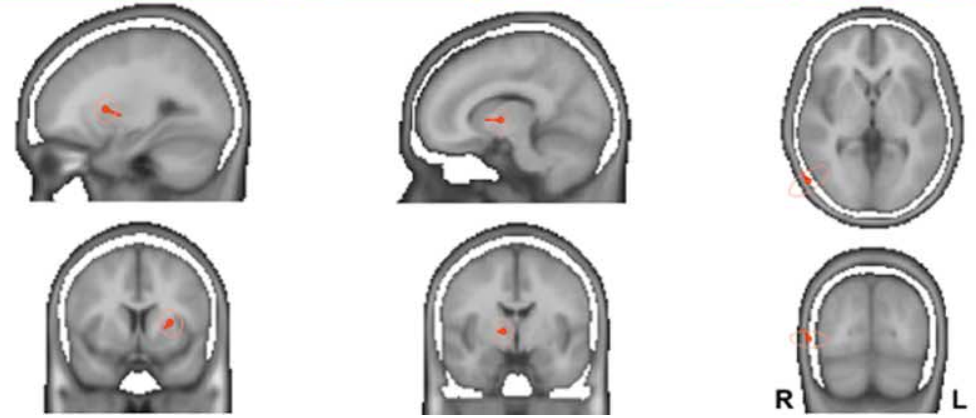

Fig. 3. (a) Representative EEG of a gelastic seizure for patient 3 (arrow signals seizure onset). There are no spikes, but a diffuse rhythmical delta activity is seen. (b) Time course of the three rhythmical ICA components obtained from the merged gelastic events. (c) ERSP for the ICA components, for three merged seizures. There is an increase in the 1-2 Hz frequency a few seconds after seizure onset, firstly in component 2 (scales as in Fig. 1). (d) Best fit dipole solution for the ICA components. A deep localization is obtained for components 2 (left) and 1 (middle), and a superficial one for component 3 (right). 
(Figs. 1(a)-3(a)). Because this seems to be a seizure related rhythm we decided to explore the possibility that it contains information on the dynamics of epileptic activity in these very peculiar seizures.

The strong association with hypothalamic hamartomas (Munari et al., 2000) and the seizure disappearence after successful removal of the lesion (Cascino et al., 1993), clearly established the hypothalamus as the brain structure more strongly associated with gelastic seizures, despite the fact that they occasionally occur in patients without lesions demonstrated in magnetic ressonance imaging (MRI) (Striano et al., 1999). The ability to extract information about the localization of the epileptic sources in gelastic seizures from scalp recordings, would be of value in the latter patients, as sometimes cortical generators are postulated, based on extrapolation from interictal spike activity (Munari et al., 1995).

Two of our patients have clearly defined hypothalamic hamartomas in MRI (Table 1), but patient 1 has only an assimetry of the position of mammilary bodies, suggesting the existence of a small expansion of the right hypothalamus. The existence of small hamartomas has been demonstrated (Striano et al., 2002), and agrees well with the work of Sherwin et al. (1962), proving that this is an anatomical area with frequent microscopic malformations.

Our ictal EEG recordings showed the usual desynchronization, movement and EMG artifacts as well as slow wave rhythmical activity (Figs. 1(a)-3(a)). The decomposition in independent components is a technique well suited to separate the contribution of artifacts from EEG activity (Makeig et al., 2004) and the much improved signal to noise ratio, resulting from their selective removal, makes the analysis of small amplitude rhythmical activity possible. In our patients, it reveals convergence of such rhythmical activity in a small number of components with distinct dipolar scalp topographies (Figs. 1(c)-3(c)). Due to the well known ill-posed character of the inverse problem in EEG, no general solution can be found to the intracranial generators of such scalp potential maps without introducing a priori properties for these generators. The dipolar distribution of the scalp potential for the components of interest in our data, suggests that a punctual source model such as the equivalent current dipole (ECD) might be appropriate and provide further insight on the dynamics of ictal epileptic activity.

Inverse source analysis of rhythmic ICA components strongly suggests the presence of deep sub-cortical sources in all patients, and cortical ones in two out of three patients. ECD models have a limited power to identify the anatomical brain structures generating scalp potential patterns and these are even more pronounced when a small number of electrodes, spherical volume conduction models and single dipole models are used. Our goal in using a simple model with all the previous handicaps was to give only a rough anatomical localization, highlighting instead the dynamics of the generators of scalp slow activity. It is one of the major goals of this work to suggest that these rhythms, up to now not studied, are an epileptic activity deserving a more detailed study with optimized source analysis methods.

The occurrence of ictal delta rhythms at consistent times after seizure onset and in different events, seems to be a replicable feature across patients and in the same patient, as is shown in Figs. 1(c)-3(c). This is also compatible with deep sources strongly attenuated and low pass filtered before their detection in scalp electrodes and agrees well with a similar trend demonstrated for interictal spikes in a previous study in patients with hypothalamic hamartoma (Leal et al., 2002). The more superficial ictal generators are located in the lobes with more consistent interictal spike activity (Table 1), which suggests a selective propagation of deep epileptic activity to particular cortical areas, frontal in patient 1 and temporal in patient 3 .

The recent improvements in structural MRI allowed the demonstration of small $\mathrm{HH}$ in cases of gelastic seizures and other brain lesions (Palmini et al., 2005) or no lesions at all (Arroyo et al., 1997). These reports shed doubt in previous cases attributing gelastic seizures to extra-hypothalamic sources (Arroyo et al., 1993) on the basis of negative findings in MRI and the demonstration of ictal sub-cortical generators in such cases can be a further reason to carefully look for small $\mathrm{HH}$.

Overall, analysis of two patients with hypothalamic hamartoma and one with a suspected hypothalamic lesion reveals the existence of ictal rhythmical activity in the scalp EEG generated in deep sub-cortical regions and with secondary involvement of frontal and temporal cortical areas.

\section{Acknowledgements}

The authors are grateful to Dr Jan de Munck, Elisa Vilar, Rita Pinto, Elisabete Lage and Bruno Mourão for technical support.

\section{Appendix A}

Decomposition in independent components was performed with the INFOMAX algorithm (Bell and Sejnowski, 1995) implemented in EEGLAB (version 4.515), using 36,000 frames to determine a number of ICA components equal to the number of electrodes $(21,32$ and 30 for patients 1,2 and 3 , respectively). The initial learning rate was 0.001 and the process stopped when weight change was smaller than 1E-7.

The ICA components with ictal rhythmic activity on visual inspection were submitted to ERSP analysis using an FFT algorithm with a sliding window of 256 points, filtered with a Hanning window and $50 \%$ overlap. To evaluate the significance of the spectral changes we used a Bootstrap method with surrogate data generated by FFT of similar 
spectral windows $(N=200)$ with onset randomized in the pre-ictal period. From the surrogate baseline distribution obtained we derived the ERSP significance level of $1 \%$ used to construct Figs. 1(c)-3(c).

Dipole fitting was done with the software Source2 (Neuroscan, El Passo), using a moving dipole in a volume conductor model of three spheres with conductivities of $0.33,0.0042$ and $0.33 \mathrm{~S} / \mathrm{m}$. Electrode positions were taken from their 10-20 standard position and the final representation done in a registered average brain. Confidence ellipsoids for dipole position were calculated using the method of Fuchs et al. (2004), for an average noise level of $10 \mu \mathrm{V}$.

\section{References}

Arroyo S, Lesser RP, Gordon B, Uematsu S, Hart J, Schwerdt P, Andreasson K, Tolosa E. Mirth, laughter and gelastic seizures. Brain 1993;116:757-80.

Arroyo S, Santamaria J, Sanmarti F, Lomenã F, Catafau A, Casamitjana R, Setoain J, Tolosa E. Ictal laughter associated with paroxysmal hypothalamopituitary dysfunction. Epilepsia 1997;38(1):114-7.

Bell AJ, Sejnowski TJ. An information-maximization approach to blind source separation and blind deconvolution. Neural Comput 1995;7: $1129-59$.

Cascino G, Andermann F, Berkovic S, Kuzniecky R, Sharbrough F, Keen D, Bladin P, Kelly P, Olivier A, Feindel W. Gelastic seizures and hypothalamic hamartomas: evaluation of patients undergoing chronic intracranial EEG monitoring and outcome of surgical treatment. Neurology 1993;43:747-50.

Delorme A, Makeig S. EEGLAB: an open source toolbox for analysis of single-trial EEG dynamics. J Neurosci Methods 2004;134:9-21.
Fuchs M, Wagner M, Kastner J. Confidence limits of dipole source reconstruction results. Clin Neurophysiol 2004;115:1442-51.

Leal AJR, Passão V, Calado E, Vieira JP, Cunha JP. Interictal spike EEG source analysis in hypothalamic hamartoma epilepsy. Clin Neurophysiol 2002;113:1961-9.

Leal AJR, Moreira A, Robalo C, Ribeiro C. Different electroclinical manifestations of the epilepsy associated with hamartomas connecting to the middle or posterior hypothalamus. Epilepsia 2003;44(9):1191-5.

List CF, Dowman CE, Bagahi BK, Bebin J. Posterior hypothalamic hamartomas and gangliomas causing precocious puberty. Neurology 1958;8:164-74.

Makeig S. Auditory event-related dynamics of the EEG spectrum and effects of exposure to tones. Electroencephalogr Clin Neurophysiol 1993;86:283-93.

Makeig S, Debener S, Onton J, Delorme A. Mining event-related brain dynamics. Trends Cogn Sci 2004;8(5):204-10.

Munari C, Kahane P, Francione S, Hoffmann D, Tassi L, Cusmai R, Vigevano F, Pasquier B, Betti O. Role of the hypothalamic hamartoma in the genesis of gelastic fits (a video-stereo-EEG study). Electroencephalogr Clin Neurophysiol 1995;95:154-60.

Munari C, Quarato P, Kahane P, Tassi L, Minotti L, Hoffmann D, Lo Russo G, Francione S, Cardinale F, Cossu M, Benabid AL. Gelastic and dacrystic seizures. In: Luders HO, Naochtar S, editors. Epileptic seizures: pathophysiology and clinical semiology. Philadelphia, PA: Churchill Livingstone; 2000.

Palmini A, Paesschen W, Dupont P, Laere K, Driel G. Status gelasticus after temporal lobectomy: ictal FDG-PET findings and the question of dual pathology involving hypothalamic hamartomas. Epilepsia 2005; 46(8):1313-6.

Sherwin RP, Grassi JE, Sommers SC. Hamartomatous malformations of the posterolateral hypothalamus. Lab Invest 1962;11:89-97.

Striano S, Meo R, Bilo L, Cirillo S, Nocerino C, Ruosi P, Striano P, Estreano A. Gelastic epilepsy: symptomatic and cryptogenic cases. Epilepsia 1999;40(3):294-302.

Striano S, Striano P, Cirillo S, Nocerino C, Bilo L, Meo R, Ruosi P, Boccela P, Briganti F. Small hypothalamic hamartomas and gelastic seizures. Epileptic Disord 2002;4:129-33. 